EPJ Web of Conferences 53, 06008 (2013)

DOI: $10.1051 /$ epjconf/20135306008

(C) Owned by the authors, published by EDP Sciences, 2013

\title{
A strategy to unveil transient sources of ultra-high-energy cosmic rays
}

\author{
Hajime Takamia ${ }^{a}$ \\ Theory Center, Institute of Particle and Nuclear Studies (IPNS), High Energy Accelerator \\ Research Organization (KEK), 1-1 Oho, Tsukuba, Ibaraki 305-0801, Japan
}

\begin{abstract}
Transient generation of ultra-high-energy cosmic rays (UHECRs) has been motivated from promising candidates of UHECR sources such as gamma-ray bursts, flares of active galactic nuclei, and newly born neutron stars and magnetars. Here we propose a strategy to unveil transient sources of UHECRs from UHECR experiments. We demonstrate that the rate of UHECR bursts and/or flares is related to the apparent number density of UHECR sources, which is the number density estimated on the assumption of steady sources, and the time-profile spread of the bursts produced by cosmic magnetic fields. The apparent number density strongly depends on UHECR energies under a given rate of the bursts, which becomes observational evidence of transient sources. It is saturated at the number density of host galaxies of UHECR sources. We also derive constraints on the UHECR burst rate and/or energy budget of UHECRs per source as a function of the apparent source number density by using models of cosmic magnetic fields. In order to obtain a precise constraint of the UHECR burst rate, high event statistics above $\sim 10^{20} \mathrm{eV}$ for evaluating the apparent source number density at the highest energies and better knowledge on cosmic magnetic fields by future observations and/or simulations to better estimate the time-profile spread of UHECR bursts are required. The estimated rate allows us to constrain transient UHECR sources by being compared with the occurrence rates of known energetic transient phenomena.
\end{abstract}

\section{INTRODUCTION}

The origin of ultra-high-energy cosmic rays (UHECRs) has been a big mystery in astrophysics over 50 years. UHECRs are mainly believed to be of extragalactic origin because their Larmor radius in the Galaxy exceeds the typical height of the Galactic disk, and various kinds of energetic astrophysical objects have been suggested as primary source candidates: gamma-ray bursts (GRBs) [1-3], active galactic nuclei (AGN) [4-10], young neutron stars or magnetars [11-15], and structure formation shocks [16-18]. Some of them are transient phenomena ${ }^{1}$.

If UHECRs with the energy of $E$ are produced in a relativistic outflow with the Lorentz factor of $\Gamma$, their sources should have isotropic luminosity

$$
L \gtrsim 2 \times 10^{45} \frac{\Gamma^{2}}{Z^{2} \beta}\left(\frac{E}{10^{20} \mathrm{eV}}\right)^{2} \quad \mathrm{erg} \mathrm{s}^{-1},
$$

where $\beta \sim 1$ is the velocity of a shock or wave in the production region in the unit of speed of light and $Z$ is the nuclear number [16, 19, 20]. If protons dominate in UHECRs as HiRes [21] and Telescope Array [22] have reported, this condition provides a severe constraint on UHECR sources; there are few astrophysical objects satisfying this condition steadily in the nearby universe. This is the case even

\footnotetext{
ae-mail: takami@post.kek.jp

${ }^{b}$ Affiliation at the moment of the conference is Max-Planck-Institute for Physics, Föhringer Ring 6, 80805 Munich, Germany

${ }^{1}$ Neutron stars and magnetars are also regarded as transient sources in the context of UHECRs because lifetime to produce UHECRs is smaller than the time-profile spread produced by cosmic magnetic fields.

This is an Open Access article distributed under the terms of the Creative Commons Attribution License 2.0, which permits unrestricted use, distribution, and reproduction in any medium, provided the original work is properly cited.
} 
for AGN correlating with the UHE events detected by the Pierre Auger Observatory (PAO) [23], although the PAO claims that correlation between UHECRs and AGN should be interpreted as that between UHECRs and matter distribution generally due to uncertainty of UHECR deflection angles by cosmic magnetic fields [24] (see also [25, 26]). However, some transient phenomena such as GRBs and AGN flares can satisfy this requirement even if their high bulk Lorentz factors are taken into account ( $\Gamma \lesssim 1000$ for GRBs and $\Gamma \gtrsim 10$ for AGN flares). Thus, there is enough motivation to discuss observational evidence of transient generation of UHECRs and how to constrain transient sources.

Here, we discuss a strategy to unveil transient UHECR sources. Although we mainly focus on proton cases contrary to the results of recent composition estimation by the PAO [27], the discussions below can apply for heavy-nuclei cases ${ }^{2}$. In Section 2 we briefly review specific aspects and observational features for transient UHECR sources. Section 3 is dedicated to consider relations among the rate of UHECR bursts, the apparent number density of UHECR sources, and cosmic magnetic fields, and to derive constraints on the rate of UHECR bursts. These discussions are based on our paper already published [31]. Then, we conclude in Section 4.

\section{OBSERVATIONAL FEATURES OF TRANSIENT UHECR SOURCES}

In the case of steady sources UHECR flux is independent of time by definition, and time-delay between the arrival time of UHECRs and that of photons emitted at the same time is not important. On the other hand, for transient cases, the time-delay plays essential roles as seen below. Let us consider the propagation of UHECRs in an isotropic turbulent magnetic field with the magnetic strength of $B$ and correlation length of $\lambda$ for simplicity. The deflection angle and time-delay are estimated as

$$
\begin{gathered}
\theta(E, D)=\frac{2 D \lambda}{9 r_{\mathrm{L}}^{2}}=2.5^{\circ} Z\left(\frac{E}{10^{20} \mathrm{eV}}\right)^{-1}\left(\frac{D}{100 \mathrm{Mpc}}\right)^{1 / 2}\left(\frac{B}{1 \mathrm{nG}}\right)\left(\frac{\lambda}{1 \mathrm{Mpc}}\right)^{1 / 2} \\
t_{\mathrm{d}}(E, D)=\frac{D \theta^{2}(E, D)}{4 c}=1.5 \times 10^{5}\left(\frac{E}{10^{20} \mathrm{eV}}\right)^{-2}\left(\frac{D}{100 \mathrm{Mpc}}\right)^{2}\left(\frac{B}{1 \mathrm{nG}}\right)^{2}\left(\frac{\lambda}{1 \mathrm{Mpc}}\right) \mathrm{yr},
\end{gathered}
$$

where $D$ is the source distance. Since the time-delay is proportional to $E^{-2}$, the arrival time of UHECRs depends on energies. As a result a UHECR spectrum from a transient source has a spiky shape. If this UHECR burst is strong and/or nearby, this spiky shape appears in the total UHECR spectrum especially above $10^{20} \mathrm{eV}$ [32].

UHECRs propagate in different trajectories due to the turbulent magnetic field and stochasticity of photomeson production, and the time-profile of a UHECR burst spreads over intrinsic burst duration. The width of the time-profile spread, $\tau(E)$, is consistent with the time-delay $t_{\mathrm{d}}$ in the case of a random magnetic field, which can be confirmed by numerical calculations. Here, remember that $t_{\mathrm{d}}(E, D)$ is also proportional to $B$ and astrophysical UHECR sources are expected to be embedded in magnetized structures such as clusters of galaxies and filamentary structures. Assuming the sizes of these structures are comparable, the time profile spread of UHECR bursts is larger in the case of sources in more strongly magnetized structures. Thus, the probability that UHECRs from a source are observed at a short time window ${ }^{3}$ is higher for sources surrounded by stronger magnetic fields, and therefore a flux reference map calculated on the assumption of steady sources (e.g., [33]) is modified [34]. This time-profile spread is regarded as the apparent duration of the UHECR burst.

Since the apparent duration of UHECR bursts also depends on UHECR energies, the number of UHECR sources contributing to the observed flux at a short time window is also dependent on energies.

\footnotetext{
${ }^{2}$ In general, deflections of heavy nuclei by cosmic magnetic fields are large (e.g., [28, 29]) and anisotropy is expected to be weaken. Possible anisotropy in the arrival distribution of UHE nuclei is recently discussed in [30].

3 "Short" means "shorter than the time-profile spread."
} 
UHECR 2012

As a result, anisotropic features in the arrival direction distribution are different between energies. This is essence of discussions in this paper, which is discussed in more details in a published paper [31].

\section{RELATION BETWEEN UHECR BURST RATE AND APPARENT SOURCE NUMBER DENSITY}

Generally the occurrence rate of rare transient $\rho_{s}$ with the duration of $\tau$ is related to the apparent source number density at a short time window by $\rho_{s} \simeq n / \tau$. For rare UHECR bursts a similar relation is valid, but the duration is replaced by an apparent duration produced by cosmic magnetic fields $\tau(E)$,

$$
\rho_{s} \simeq \frac{n_{s}(E)}{\tau(E)},
$$

where $n_{s}(E)$ is the apparent number density of UHECR sources. $n_{s}(E)$ can be estimated from anisotropy measurements of detected events on the assumption of steady sources [35, 36], as long as the observation term is shorter than $\tau(E)$.

Equation (4) is valid only when each UHECR burst can be individually identified as a burst spatially and temporally [37]. If more than one bursts or flares occurring in an angular patch contribute to UHECRs observed in the same time-window, i.e., the time profiles of two independent UHECR bursts from the same direction within the size of the angular patch are overlapped at the Earth, equation (4) cannot be used as it is. Since UHECRs have finite deviation angles due to cosmic magnetic fields, UHECRs from a source arrive within a finite solid angle $\Delta \Omega=\pi \psi^{2}$ around the source, which can be regarded as the appropriate size of the finite angular patch. For a given $\rho_{s}$ the typical time interval between bursts in the direction with $\Delta \Omega$ is

$$
\Delta T \sim \frac{3}{\Delta \Omega D_{\max }(E)^{3} \rho_{s}} \sim 3 \times 10^{5}\left(\frac{\psi}{5^{\circ}}\right)^{-2}\left(\frac{\rho_{s}}{1 \mathrm{Gpc}^{-3} \mathrm{yr}^{-1}}\right)^{-1}\left(\frac{D_{\max }(E)}{75 \mathrm{Mpc}}\right)^{-3} \quad \mathrm{yr},
$$

where $D_{\max }(E)$ is the maximum distance of UHECR sources which can contribute to the UHECR flux with energies $E$. Here we set $\psi \sim 5^{\circ}$ as a reference choice. Since equation (4) is valid if $\tau(E)<\Delta T$, i.e.,

$$
n_{s}(E) \lesssim 3 \times 10^{-4}\left(\frac{\psi}{5^{\circ}}\right)^{-2}\left(\frac{D_{\max }(E)}{75 \mathrm{Mpc}}\right)^{-3} \mathrm{Mpc}^{-3} .
$$

Otherwise, $\tau(E)>\Delta T$ provides a direct constraint to UHECR burst rate $\rho_{s}$. Thus,

$$
\rho_{s} \gtrsim 3 \times 10^{5} \frac{1}{\tau(E)}\left(\frac{\psi}{5^{\circ}}\right)^{-2}\left(\frac{D_{\max }(E)}{75 \mathrm{Mpc}}\right)^{-3} \mathrm{Gpc}^{-3} \mathrm{yr}^{-1} .
$$

Let us consider how anisotropic features in the UHECR arrival distribution are found as a function of UHECR energies. $n_{s}(E)$ is used for an indicator of the anisotropy. The left panel of figure 1 demonstrates the dependence of $n_{S}(E)$ on energies under two given $\rho_{s}$ from equation (4). In order to estimate $\tau(E)$ an effective turbulent magnetic field model with $B \lambda^{1 / 2}=0.3 \mathrm{nG} \mathrm{Mpc}^{-1}$ derived from the assumption that all the UHECR sources are embedded in filamentary structures is adopted. The panel is divided into three regions; equation (4) cannot be adopted, i.e., $\tau>\delta T$, in the upper left region, equation (4) is valid in the middle region, and no UHECR bursts in $D_{\max }(E)$ in the lower right region. Thus, $\rho_{s}$ can be estimated only in the middle phase and blue dotted lines represent $n_{S}(E)$ for $\rho=1 \mathrm{Gpc}^{-3} \mathrm{yr}^{-1}$ and $\rho=100 \mathrm{Gpc}^{-3} \mathrm{yr}^{-1}$, respectively.

$n_{s}(E)$ sensitively depends on the energy of UHECRs observed at the Earth, which is a characteristic feature of transient sources. The other feature is the saturation of $n_{s}(E)$ at lower energies. This is caused by the fact that the apparent source number density cannot exceed the number density of host galaxies of UHECR sources $n_{h}$. Here, $n_{h}=10^{-4} \mathrm{Mpc}^{-3}$ is assumed for a demonstrative purpose, which is comparable with the number density of Fanaroff-Riley I galaxies [38]. Thus, the saturated number 

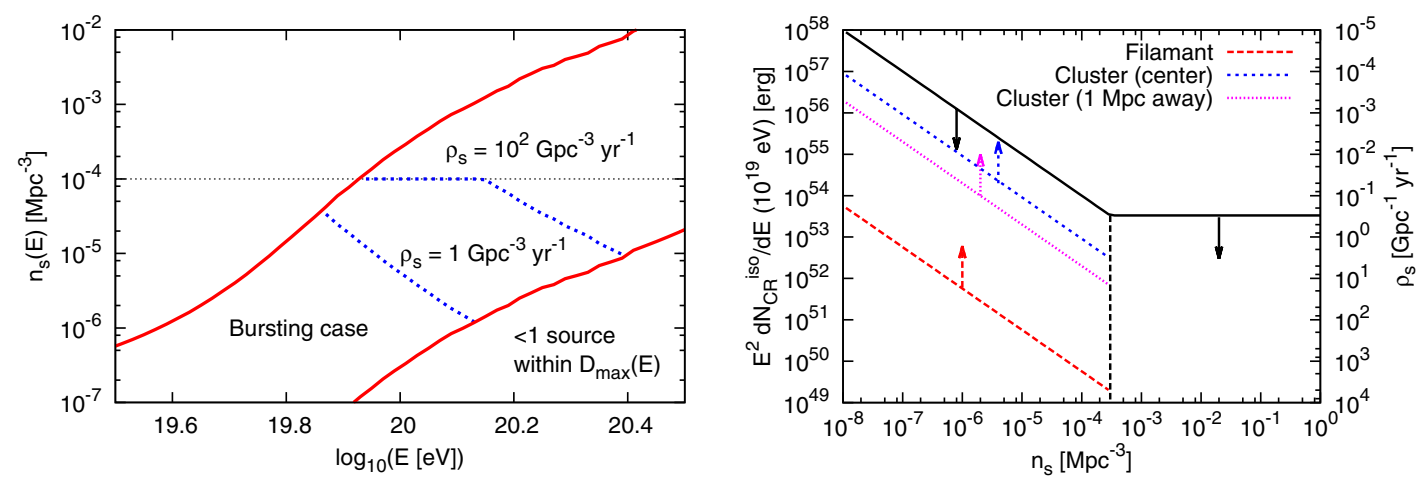

Figure 1. Left: apparent source number density $n_{s}(E)$ of UHECRs under given rates of UHECR bursts $\rho_{s}$ as a function of energy $E$ (blue). There are three regions separated by red lines in the panel; equation (4) cannot be adopted in the upper left region, equation (4) is valid in the middle region, and no UHECR sources in the lower right region. $n_{s}(E)$ is saturated at the number density of host galaxies of UHECR sources, which is assumed to be $10^{-4} \mathrm{Mpc}^{-3}$ for a demonstrative purpose. $\psi=5^{\circ}$ and $B_{\text {eff }} \lambda_{\text {eff }}^{1 / 2}=0.3 \mathrm{nG} \mathrm{Mpc} c^{1 / 2}$ are assumed. Right: constraints on the rate of UHECR bursts or flares $\rho_{s}$ and the (differential) energy budget of UHECRs per burst at $10^{19} \mathrm{eV}$ as a function of apparent source number density $n_{s}(E)$ estimated at $E=10^{20} \mathrm{eV}$, which are derived on the assumption that UHECR sources are embedded in filamentary structures (red) and clusters of galaxies (blue and magenta). For the latter case two positions are considered: the center of the clusters (blue) and $1 \mathrm{Mpc}$ away from the center (magenta). Black solid lines are the lower limits of $\rho_{s}$ derived from maximal intergalactic magnetic fields in voids. The vertical dashed line represents the border where equation (4) is valid or not, which corresponds to the upper red line in the right panel. $\psi=5^{\circ}$ is assumed.

density is another information to constrain UHECR sources by being compared with the number density of known specific galaxies. Note that a number of the highest energy events $\left(\gtrsim 10^{20} \mathrm{eV}\right)$ are required to identify the dependence of $n_{s}(E)$ on $E$. Although the number should be estimated in details in future studies, $\sim 100$ events above $10^{20} \mathrm{eV}$ may be required for the first step to estimate $n_{s}(E)$ at two energy bands in the middle region.

Next, let us derive constraints on UHECR burst rate $\rho_{s}$. We consider a simple three zone cosmic magnetic field model; the first is a magnetic field surrounding UHECR sources such as a filament or cluster of galaxies, the second is intergalactic magnetic fields (IGMFs) in voids, and the third is the Galactic magnetic field (GMF). We calculate the propagation of protons in these magnetic structures and estimate the time-profile spread of a UHECR burst.

The strength of a magnetic field in a cluster of galaxies is assumed to scale with the distance from the center of the cluster following the model in Ref. [39]. The strength is normalized as $1 \mu \mathrm{G}$ at the center. The direction of the magnetic field is set to be turbulent with the Kolmogorov power spectrum with the maximum scale of $\lambda_{\mathrm{c}, \max }=400 \mathrm{kpc}$ to reproduce the deflection angles of UHECRs estimated from the analytical estimation, i.e., equation (2) for $\lambda=100 \mathrm{kpc}$. A filamentary structure is modeled as a cylinder with the radius of $2 \mathrm{Mpc}$, a turbulent magnetic field with the Kolmogorov power spectrum with $B=10 \mathrm{nG}[40]$ and $\lambda_{\mathrm{c}, \max }=400 \mathrm{kpc}$. A bisymmetric spiral field model with even parity is assumed for the GMF [41].

IGMFs are highly uncertain. Faraday rotation measurements provides an upper limit of $B \lambda^{1 / 2} \lesssim$ $(10 \mathrm{nG})(1 \mathrm{Mpc})^{1 / 2}$ (e.g., [42]) and cosmology gives $B<2.5 \mathrm{nG}$ for $\lambda=1 \mathrm{Mpc}$ (e.g., [43]). On the other hand, recent studies on TeV blazars with hard spectra imply $B \gtrsim 10^{-18} \mathrm{G}$ (e.g., [44]). In this range the time-delay by IGMFs dominate over that by other magnetic fields in some cases and vice versa in the other cases. Thus, we constrain $\rho_{s}$ with the uncertainty of this range. Magnetic fields embedding UHECR sources and the GMF inevitably affect the propagation of UHECRs, and therefore the minimum of the time-profile spread is defined as $\tau_{\min }(E)$. The maximum of the time-profile spread is $\tau_{\max }(E)$ by 
UHECR 2012

Table 1. Potential transient sources and their typical local rates [37].

\begin{tabular}{lcc}
\hline Source & Typical rate $\left[\mathrm{Gpc}^{-3} \mathrm{yr}^{-1}\right]$ & Reference \\
\hline HL GRB & $\sim 0.1$ & e.g., [49] \\
LL GRB & $\sim 400$ & e.g., [50] \\
Hypernova & $\sim 2000$ & e.g., [51] \\
Magnetar & $\sim 12000$ & e.g., [52] \\
Giant Magnetar Flare & $\sim 10000$ & e.g., [53] \\
Giant AGN Flare & $\sim 1000$ & [6] \\
Supernova (SN) Ibc & $\sim 20000$ & e.g., [51] \\
Core Collapse SN & $\sim 120000$ & e.g., [54] \\
\hline
\end{tabular}

adding maximal contribution of intergalactic magnetic fields to $\tau_{\min }(E)$. Then, a constraint on $\rho_{s}$ can be written as

$$
\frac{n_{s}(E)}{\tau_{\max }(E)} \lesssim \rho_{s} \lesssim \frac{n_{s}(E)}{\tau_{\min }(E)}
$$

in the regime in which equation (4) is valid.

The observed UHECR flux indicates that the energy budget of UHECRs per volume at $10^{19} \mathrm{eV}$ is $\mathcal{E}_{\mathrm{CR}} \sim 10^{44} \mathrm{erg} \mathrm{Mpc}^{-3} \mathrm{yr}^{-1}[37,45,46]$. This means that UHECR energy budget per source is

$$
E^{2} \frac{d N}{d E}(E) \sim \frac{\mathcal{E}_{\mathrm{CR}}}{\rho_{s}} \sim 10^{53}\left(\frac{\rho_{s}}{1 \mathrm{Gpc}^{-3} \mathrm{yr}^{-1}}\right)^{-1} \quad \text { erg. }
$$

Thus, constraints on $\rho_{s}$ is equivalent to constraints on the energy budget.

The right panel of Figure 1 shows constraints on $\rho_{s}$ and the energy budget of UHECRs with $10^{19} \mathrm{eV}$ per burst as a function of $n_{s}(E)$ at $E=10^{20} \mathrm{eV}$, which are derived on the assumption that UHECR sources are embedded in filamentary structures or clusters of galaxies. At present the apparent source number density best to reproduce the recent PAO data is $\sim 10^{-4} \mathrm{Mpc}^{-3}[35,36]$, but we should keep in mind that this value is estimated at around $\sim 6 \times 10^{19} \mathrm{eV}$ because of the steep spectrum of UHECRs. $n_{S}(E)$ is expected to be smaller at higher energies from the discussions for the left panel of Figure 1. $n_{s}(E)$ should be determined by future gigantic UHECR experiments such as Extreme Universe Space Observatory on broad Japanese Experiment Module (JEM-EUSO) [47].

Once the possible range of $\rho_{s}$ (and the energy budget of UHECRs per source) is estimated, one can constrain transient UHECR source candidates by comparing the derived $\rho_{s}\left(E^{2} d N / d E\right)$ and the occurrence rates (energy input) of known energetic transient phenomena, listed in Table 1.

Although models are used, better knowledge on structured and intergalactic magnetic fields is important for more reliable constraints. Future all-sky Faraday rotation measurements by radio observations such as Square Kilometer Array [48] and detailed simulations help our understanding of $\tau(E)$.

\section{CONCLUSION}

We have proposed and demonstrated a strategy to unveil transient sources of UHECRs from UHECR experiments. High event statistics with $E \gtrsim 10^{20} \mathrm{eV}(\sim 100$ events for a first step, although this number should be studied more quantitatively) is essential for this purpose because the highest energy cosmic rays arrive at the Earth only from small nearby universe. Strong dependence of the apparent source number density $n_{s}(E)$ on UHECR energies $E$ provides evidence of transient generation of UHECRs. The number density is saturated at that of host galaxies of UHECR sources, which is another hint for UHECR sources via connection between UHECR source candidates and host galaxies. The apparent source number density $n_{s}(E)$ and the time-profile spread of UHECR bursts or flares $\tau(E)$ estimated 
from good information on cosmic magnetic fields allow us to constrain a UHECR burst rate $\rho_{s}$. This rate enables us to constrain transient UHECR sources by being compared with the occurrence rates of known energetic transient phenomena. $n_{S}(E)$ can be well estimated from data taken by future gigantic UHECR experiments such as JEM-EUSO. For precise estimation of $\tau(E)$ good knowledge on the structured EGMFs from observations and or simulations is required.

H.T. thanks Kohta Murase for fruitful discussions. The work of H.T. is supported by Japan Society for the Promotion of Science (JSPS) via JSPS fellowship.

\section{References}

[1] E. Waxman, Phys. Rev. Lett. 75, 386 (1995)

[2] M. Vietri, Astrophys. J. 453, 883 (1995)

[3] K. Murase, K. Ioka, S. Nagataki, T. Nakamura, Phys. Rev. D 78, 023005 (2008)

[4] P.L. Biermann, P.A. Strittmatter, Astrophys. J. 322, 643 (1987)

[5] F. Takahara, Prog. Theor. Phys. 83, 1071 (1990)

[6] G.R. Farrar, A. Gruzinov, Astrophys. J. 693, 329 (2009)

[7] C.D. Dermer, S. Razzaque, J.D. Finke, A. Atoyan, New Journal of Physics 11, 065016 (2009)

[8] A. Pe'er, K. Murase, P. Meszaros, Phys. Rev. D 80, 123018 (2009)

[9] H. Takami, S. Horiuchi, Astroparticle Physics 34, 749 (2011)

[10] K. Murase, C.D. Dermer, H. Takami, G. Migliori, Astrophys. J. 749, 63 (2012)

[11] P. Blasi, R.I. Epstein, A.V. Olinto, Astrophys. J. 533, L123 (2000)

[12] J. Arons, Astrophys. J. 589, 871 (2003)

[13] K. Murase, P. Meszaros, B. Zhang, Phys. Rev. D 79, 103001 (2009)

[14] K. Kotera, Phys. Rev. D 84, 023002 (2011)

[15] K. Fang, K. Kotera, A.V. Olinto, Astrophys. J. 750, 118 (2012)

[16] C.A. Norman, D.B. Melrose, A. Achterberg, Astrophys. J. 454, 60 (1995)

[17] H. Kang, D. Ryu, T.W. Jones, Astrophys. J. 456, 422 (1996)

[18] S. Inoue, G. Sigl, F. Miniati, E. Armengaud (2007), astro-ph/0701167

[19] R.D. Blandford, Phys. Scr. T 85, 191 (2000)

[20] E. Waxman, Pramana 62, 483 (2004)

[21] R.U. Abbasi et al., Phys. Rev. Lett. 104, 161101 (2010)

[22] Y. Tameda, proceedings of this conference

[23] I. Zaw, G.R. Farrar, J.E. Greene, Astrophys. J. 696, 1218 (2009)

[24] J. Abraham et al., Science 318, 938 (2007)

[25] H. Takami, T. Nishimichi, K. Yahata, K. Sato, J. Cosmo. Astropart. Phys. 0906, 031 (2009)

[26] H. Takami, T. Nishimichi, K. Sato, Prog. Theor. Phys. 126, 1123 (2011)

[27] J. Abraham, P. Abreu, M. Aglietta, E.J. Ahn, D. Allard, et al., Phys. Rev. Lett. 104, 091101 (2010)

[28] H. Takami, K. Sato, Astrophys. J. 724, 1456 (2010)

[29] G. Giacinti, M. Kachelrieß, D.V. Semikoz, G. Sigl, J. Cosmo. Astropart. Phys. 8, 036 (2010)

[30] H. Takami, S. Inoue, T. Yamamoto, Astropart. Physics 35, 767 (2012)

[31] H. Takami, K. Murase, Astrophys. J. 748, 9 (2012)

[32] J. Miralda-Escude, E. Waxman, Astrophys. J. 462, L59 (1996)

[33] H.B.J. Koers, P. Tinyakov, MNRAS 399, 1005 (2009)

[34] S. Kalli, M. Lemoine, K. Kotera, Astron. \& Astrophys. 528, A109 (2011)

[35] H. Takami, K. Sato, Astropart. Phys. 30, 306 (2009)

[36] A. Cuoco, S. Hannestad, T. Haugbølle, M. Kachelrieß, P.D. Serpico, Astrophys. J. 702, 825 (2009)

[37] K. Murase, H. Takami, Astrophys. J. Lett. 690, L14 (2009)

[38] P. Padovani, C.M. Urry, Astrophys. J. 356, 75 (1990) 


\section{UHECR 2012}

[39] D. de Marco, P. Hansen, T. Stanev, P. Blasi, Phys. Rev. D 73, 043004 (2006)

[40] D. Ryu, H. Kang, J. Cho, S. Das, Science 320, 909 (2008)

[41] J. Alvarez-Mu niz, R. Engel, T. Stanev, Astrophys. J. 572, 185 (2002)

[42] P. Blasi, S. Burles, A.V. Olinto, Astrophys. J. Lett. 514, L79 (1999)

[43] D.G. Yamazaki, K. Ichiki, T. Kajino, G.J. Mathews, Phys. Rev. D 81, 023008 (2010)

[44] C.D. Dermer, M. Cavadini, S. Razzaque, J.D. Finke, J. Chiang, B. Lott, Astrophys. J. Lett. 733, L21 (2011)

[45] E. Waxman, J.N. Bahcall, Phys. Rev. D 59, 023002 (1999)

[46] V. Berezinsky, A. Gazizov, S. Grigorieva, Phys. Rev. D 74, 043005 (2006)

[47] T. Ebisuzaki, Y. Uehara, H. Ohmori, K. Kawai, et al., Nucl. Phys. B Proc. Suppl. 175, 237 (2008)

[48] http://www.skatelescope.org/

[49] D. Guetta, T. Piran, J. Cosmo. Astropart. Phys. 7, 3 (2007)

[50] E. Liang, B. Zhang, F. Virgili, Z.G. Dai, Astrophys. J. 662, 1111 (2007)

[51] D. Guetta, M. Della Valle, Astrophys. J. Lett. 657, L73 (2007)

[52] B.M. Gaensler, N.M. McClure-Griffiths, M.S. Oey, M. Haverkorn, et al., Astrophys. J. Lett. 620, L95 (2005)

[53] E.O. Ofek, Astrophys. J. 659, 339 (2007)

[54] P. Madau, M. della Valle, N. Panagia, MNRAS 297, L17 (1998) 